\title{
Excretion of purine derivatives in cows: endogenous contribution and recovery of exogenous purine bases
}

\begin{abstract}
Two dry cows fitted with simple cannula in the rumen and duodenum, and fed with a mixed diet (straw:barley, 50:50), were used to determine endogenous losses and response of urinary purine derivatives (PDs) to duodenally infused yeast-RNA. Duodenal flow of purine bases (PBs) was determined by a dual marker system, and $15 \mathrm{~N}$ was infused continuously into the rumen to label microbial PBs. The isotope dilution of urinary PDs in relation to duodenal PBs confirmed the presence of an endogenous fraction (236 عmol/kg LW0.75) bigger than in sheep and lower than values estimated in cows with impaired rumen fermentation. Excretion of PDs increased linearly in response to incremental supply of PBs with an equimolar recovery of $0.84 \mathrm{~mol} / \mathrm{mol}$. However, net recovery of duodenal PBs was 0.67 for the basal diet and $0.65,0.90,0.79$ and 0.82 for the four levels of PB infusion. It is suggested that differences in digestibility between yeast-RNA and duodenal PBs might explain differences in recovery estimations.
\end{abstract}

Keyword: Cattle ï feeding and nutrition; Nucleic acid; Purine derivatives; Allantoin 